\title{
Rapid Prototyping of Electrically Small Spherical Wire Antennas
}

\author{
Kim, Oleksiy S.
}

Published in:

I E E E Transactions on Antennas and Propagation

Link to article, DOI:

10.1109/TAP.2014.2317489

Publication date:

2014

Document Version

Early version, also known as pre-print

Link back to DTU Orbit

Citation (APA):

Kim, O. S. (2014). Rapid Prototyping of Electrically Small Spherical Wire Antennas. I E E E Transactions on Antennas and Propagation, 62(7), 3839-3842. https://doi.org/10.1109/TAP.2014.2317489

\section{General rights}

Copyright and moral rights for the publications made accessible in the public portal are retained by the authors and/or other copyright owners and it is a condition of accessing publications that users recognise and abide by the legal requirements associated with these rights.

- Users may download and print one copy of any publication from the public portal for the purpose of private study or research.

- You may not further distribute the material or use it for any profit-making activity or commercial gain

- You may freely distribute the URL identifying the publication in the public portal

If you believe that this document breaches copyright please contact us providing details, and we will remove access to the work immediately and investigate your claim. 


\section{Rapid prototyping of electrically small spherical wire antennas}

\author{
Oleksiy S. Kim
}

\begin{abstract}
It is shown how modern rapid prototyping technologies can be applied for quick and inexpensive, but still accurate, fabrication of electrically small wire antennas. A well known folded spherical helix antenna and a novel spherical zigzag antenna have been fabricated and tested, exhibiting the impedance and radiation characteristics in close agreement with those predicted numerically.
\end{abstract}

Index Terms-electrically small antennas, spherical antennas, rapid prototyping, 3D printing, selective laser sintering, nanocrystaline copper

\section{INTRODUCTION}

A spherical antenna is one of the fundamental concepts in the theory of electrically small antennas. The concept was introduced by $\mathrm{Chu}$ in [1], where he considered an imaginary sphere of radius $a$ with no fields inside (except those needed to make an antenna self-resonant). By evaluating the stored energy external to the sphere, he derived a lower bound on the radiation quality factor $Q$, which is expressed as [2]

$$
Q_{\mathrm{Chu}}=\frac{1}{(k a)^{3}}+\frac{1}{(k a)}
$$

where $k$ is the free space propagation constant.

Subsequently, lower bounds for $Q$ were derived for vanishingly small spherical antennas with magnetodielectric cores [3], for arbitrary size spherical antennas with air core [4], for arbitrary size spherical magnetic dipole antennas with solid magnetodielectric cores [5], as well as it was shown that the Chu lower bound could be approached by both electric and magnetic dipole spherical antennas of arbitrary size using metal cores coated with a highly permeable magnetic material [6], [7]. The latter two combined in a dual-mode configuration form an antenna that is self-resonant with the $Q$ approximately half of the Chu lower bound [8].

Numerous practical configurations of spherical antennas have been proposed and tested against the bounds, e.g. [9]-[11]. Most of these antennas were of wire type, and it was natural to try to fabricate them using conductig wires. This required substantial skills in handwork, while satisfactory results were not guaranteed. Moreover, the repetability was very poor.

More controllable methods include printing on planar [10] and curved [12], [13] substrates, direct transfer patterning [14], and even injection of liquid metal alloy into an inflatable substrate [15]. All these techniques rely on sophisticated technology and equipment, which is not readily available; and they require some kind of dielectric substrate, which always increases the $Q$ [16].

On the other hand, the recent decade's explosive growth of rapid prototyping (RP) techniques has made them available to

O. S. Kim is with the Department of Electrical Engineering, Section for Electromagnetics, Technical University of Denmark, Kgs. Lyngby, Denmark e-mail: osk@elektro.dtu.dk.

Manuscript received December xx, 2013; revised February xx, 2014. an ordinary consumer. These are stereolithography (SLA), selective laser sintering (SLS), three-dimensional printing (3DP), fused deposition modeling (FDM) just to mention a few [17]. Nowadays, RP technology is not limited to prototype building; it is also widely used for fabrication of final products and its parts.

In this paper, it is shown how a rapid prototyping technique can be applied for fabrication of electrically small spherical wire antennas. The approach is exemplified by a well-known folded spherical helix antenna and a novel spherical zigzag antenna; both are of the electric dipole type. Printed in dielectric, the antennas are subsequently coated with a conductive layer. A good conductivity of the latter is crititcal for achieving a high radiation efficiency of electrically small antennas, as discussed in Section II. The measured impedance and radiation characteristics of the fabricated antennas are presented in Section III. Concluding remarks are given in Section IV.

\section{Antennas to Print}

Among a variety of configurations, two spherical wire antennas have been selected for testing the RP technology. A folded spherical helix antenna was selected as the most well-known spherical wire antenna, which is often used as a reference for new antenna designs as well as a test object for new fabrication techniques. The second antenna was a spherical zigzag antenna, a modification of a spherical meander antenna first presented in [18]. The design needed experimental validation and the RP testing offered such an opportunity.

1) Folded Spherical Helix Antenna: consists of a number of identical wire helices wound on a spherical surface of radius $r_{0}$ [9]. One of the helices is attached, through a metal ground plane, to the inner conductor of a coaxial feeding cable. The resonance frequency is adjusted by the number of turns in the helices, whereas the input resistance at the resonance takes discrete values with changing the number of helices. The radius of the antenna was selected to be $r_{0}=25 \mathrm{~mm}$ with the radius of the wires $w=0.64 \mathrm{~mm}$. A configuration of four helices with 0.95 turns in each exhibits $50 \mathrm{ohms}$ input impedance at the resonance frequency of $750 \mathrm{MHz}$. The resulting electrical size of the antenna is $k a=k\left(r_{0}+w\right)=0.4$.

2) Spherical Zigzag Antenna: similar to the spherical meander antenna [18] is composed of wire rings successively interconnected by wire arcs, see Fig. 1. However, in contrast to the spherical meander antenna, in which the rings are parallel to each other, the spherical zigzag antenna has its rings tilted. The angle between two neighbor rings $\Delta \gamma$ is a parameter, which is used to tune the antenna to a desired resonance frequency. The radius of the $i$-th ring can be determined as

$$
r_{i}=0.5 r_{0} \sqrt{2\left(1+\cos \left(\gamma_{i}+\gamma_{i+1}\right)\right)},
$$

where $\gamma_{i}$ and $\gamma_{i+1}, \gamma_{i+1}-\gamma_{i}=\Delta \gamma$, are the elevation angles for the bottom and top tips of the $i$-th ring, respectively. The antenna is made of the same wires $(w=0.64 \mathrm{~mm})$ and has the same radius $\left(r_{0}=25 \mathrm{~mm}\right)$ as the folded spherical helix antenna described above (Section II-1). With five rings and the angle $\Delta \gamma=10.75^{\circ}$ between them, it exhibits the resonance at $750 \mathrm{MHz}$. The input resistance is fine tuned to $50 \mathrm{ohms}$ by two 


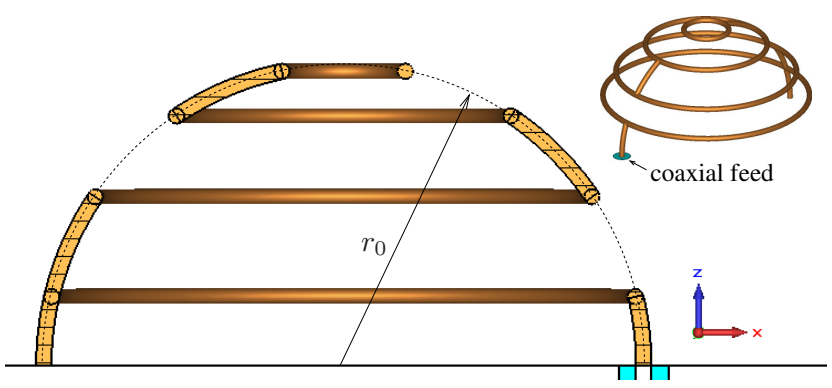

(a)

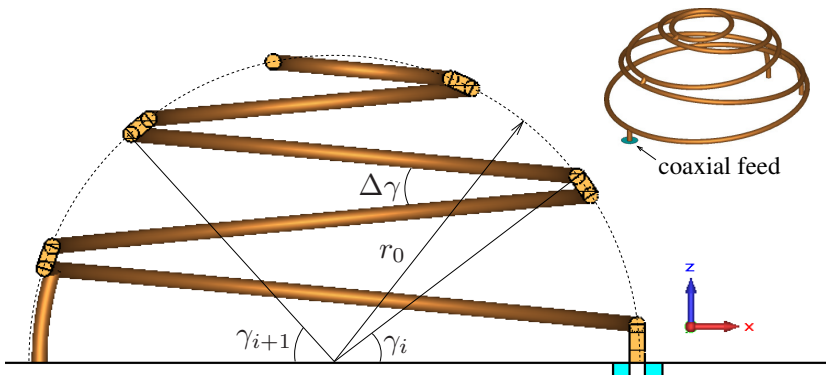

(b)

Fig. 1. Cross sections of spherical meander antenna (a) and spherical zigzag antenna (b), both fed by a coaxial cable through a ground plane. A 3D view of each antenna is shown in the respective inset.

shorting pins placed symmetrically opposite to the excitation pin, in a way described in [18].

Tilting of the rings affects negligibly the far-field radiation; the radiation pattern of the spherical meander and zigzag antennas is that of an electrically small electric dipole (or monopole, in a ground plane is utilized) with the cross-polarization (according to Ludwig's second definition [19]) generated by the $\mathrm{TE}_{11}$ spherical mode excited by the antennas. The crosspolarization is zero in the XZ-plane (Fig. 2b), because this is a symmetry plane for the antenna geometry. This property distinguishes the spherical meander and zigzag antennas from the folded spherical helix antenna, whose cross-polarization is omnidirectional (Fig. 2a).

Tilted rings give the zigzag antenna a clear advantage over its meander counterpart in terms of the radiation efficiency, when the conductivity of the wires, the antennas are made of, is finite. This is illustrated in Fig. 3, where the radiation efficiency computed with CST is plotted for the spherical meander and zigzag antennas, as well as for the folded spherical helix antenna, versus the conductivity of the wires. As the conductivity decreases, the radiation efficiency drops for all antennas, but at different rates. The degradation is slowest for the folded spherical helix antenna, while the fastest deterioration of the efficiency occurs for the spherical zigzag antenna. Nevertheless, for good conductivity, the spherical zigzag antenna outperforms not only the spherical meander antenna, but also the folded spherical helix antenna, when the conductivity approaches the value for copper. The reason is that electric currents on all parts of the zigzag antenna - including the tilted rings - contribute to the far-field radiation, whereas in the meander antenna, the far-field is due to the vertical segments, while the parallel rings contribute mainly to the reactive near-field, leading to increased losses.

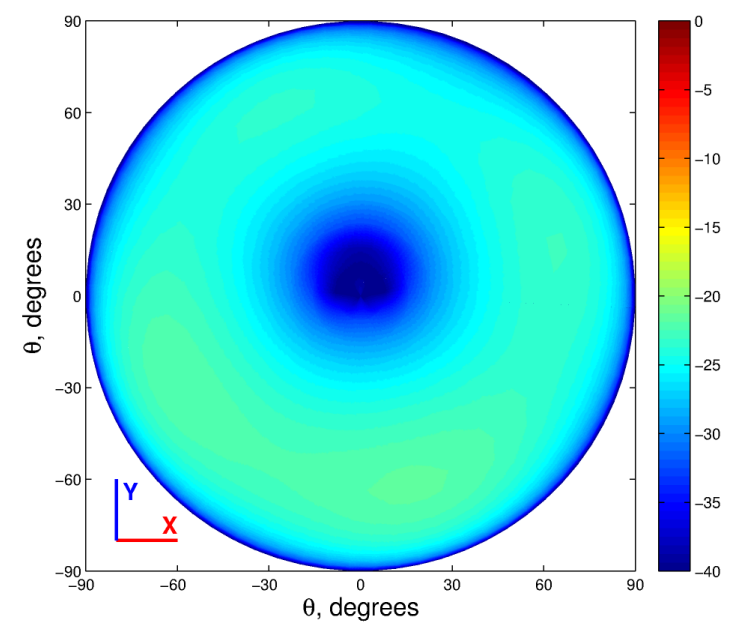

(a)

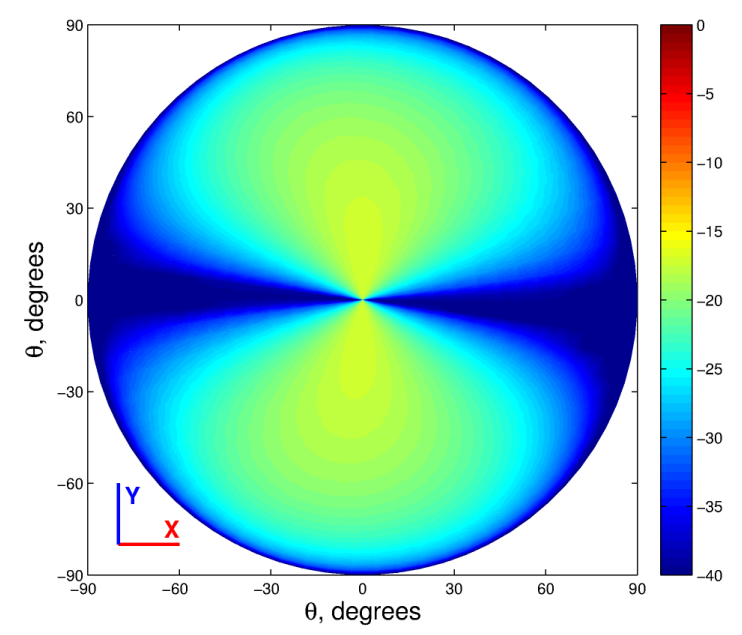

(b)

Fig. 2. Cross-polarization radiation pattern of the folded spherical helix antenna (a), and the spherical meander and zigzag antennas (b).

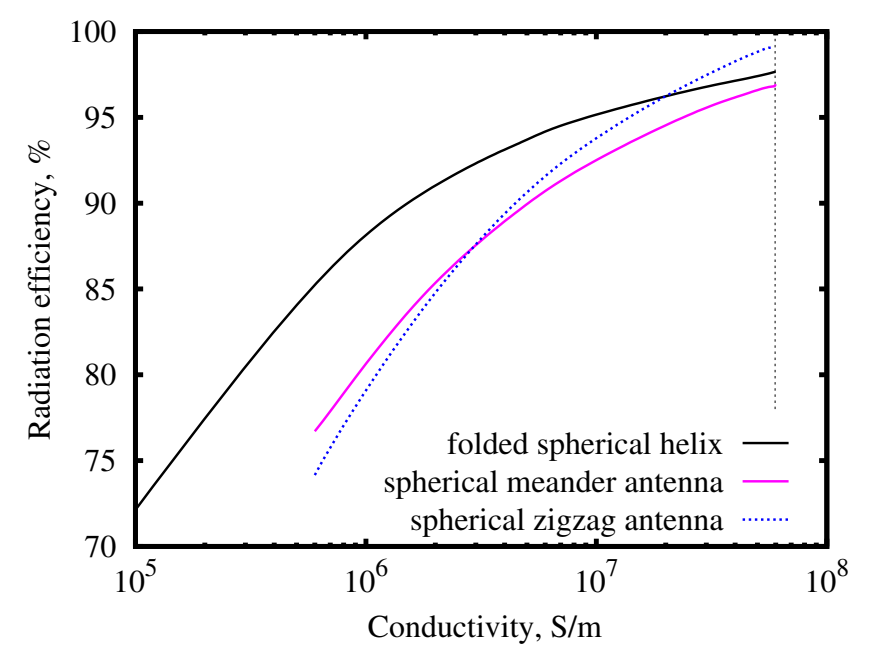

Fig. 3. Computed radiation efficiency versus the conductivity of the wires. The conductivity for copper $\left(5.96 \cdot 10^{7} \mathrm{~S} / \mathrm{m}\right)$ is marked by a vertical dotted line.

This result, besides confirming a high sensitivity of electrically small antennas to conductive losses, shows that in different ranges of the attained conductivity, different antennas 
perform better than others. When moderate to good conductivity (better than $3 \cdot 10^{6} \mathrm{~S} / \mathrm{m}$ ) is expected to be achieved in fabrication, the folded spherical helix antenna and the spherical zigzag antenna are the antennas of choice.

\section{FAbrication AND Measurements}

A solid CAD model for each of the antennas to be printed using SLS technique consisted of the corresponding wire structure and a solid cylindrical pedestal with a hole for an SMA connector. To counteract gravity induced deformations experienced during the first attempt of fabricating the folded spherical helix antenna with an RP technology [20], the wire structure was reinforced with thin supporting arcs.

Printed in dielectric, the antenna prototypes required conductive coating. In [20], it was done with copper paint; however, the results were not satisfactory, as measurements showed radiation efficiency of only $80 \%$. Therefore, an alternative conductive coating technique was utilized - coating with polycrystalline copper. By this technique, conductivities comparable to bulk copper of the corresponding thickness can be achieved on a dielectric surface, or on a part of it, as the coating can be deposited selectively. Such conductivity is about two orders of magnitude better than that of the copper paint used in [20]. The process is relatively cheap and has been already commercialized. The entire structure including the pedestal, but excluding the supporting arcs, was coated in both antenna prototypes. The central pin of an SMA connector was attached to the wire structure with a conductive glue. Finally, the printed and coated structures were embedded in a circular aluminum ground plane of $770 \mathrm{~mm}$ in diameter. The resulting prototypes of the folded spherical helix and spherical zigzag antennas are shown in Fig. 4.

Input impedance measurements showed that both antennas were well matched in vicinity of the expected resonance frequency of $750 \mathrm{MHz}$ (Fig. 5). The radiation efficiency at the resonance, measured by the Wheeler cap method, is $94 \%$ and $90 \%$ for the folded spherical helix antenna and spherical zigzag antenna, respectively ${ }^{1}$. Although the obtained efficiency is much better than that of the first prototype coated with copper paint [20], it still does not reach the maximum values predicted for the antennas made of solid copper wires (Fig. 5), indicating that the efficiency can be further improved by increasing the thickness and/or conductivity of the conductive coating.

The radiation measurements were carried out at the DTUESA Spherical Near-Field Antenna Test Facility. In particular, the interest was to see if the antennas exhibited the expected cross-polarization radiation patterns. It is observed in Fig. 6a and $6 \mathrm{~b}$, where the measured cross-polarization component of the respective radiation patterns for the folded spherical helix antenna and spherical zigzag antenna are plotted, that the crosspolarization for the former is omnidirectional, whereas the latter yields a null in the XZ-plane, in full agreement with the predictions (Fig. 2).

${ }^{1}$ These efficiencies can be obtained in simulations for antennas made of solid metal wires with conductivity of about $5 \cdot 10^{6} \mathrm{~S} / \mathrm{m}$ (Fig. 3). In this region of conductivities, the spherical helix antenna is more efficient than the spherical zigzag antenna.

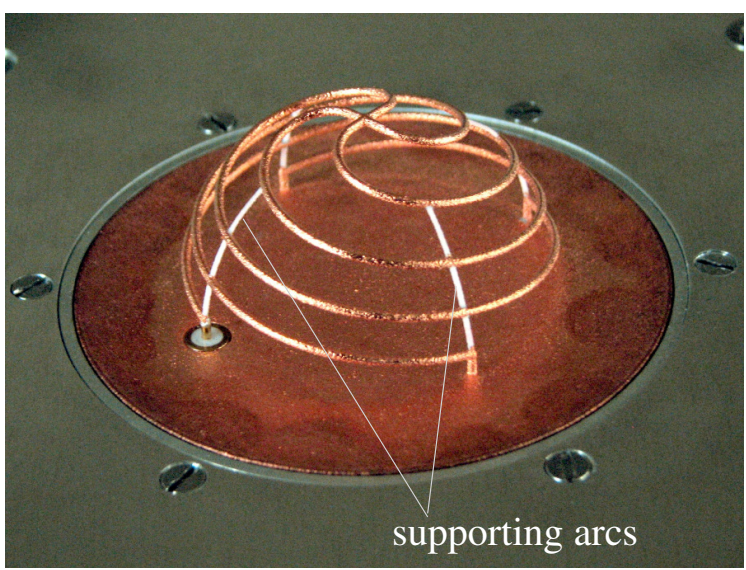

(a)

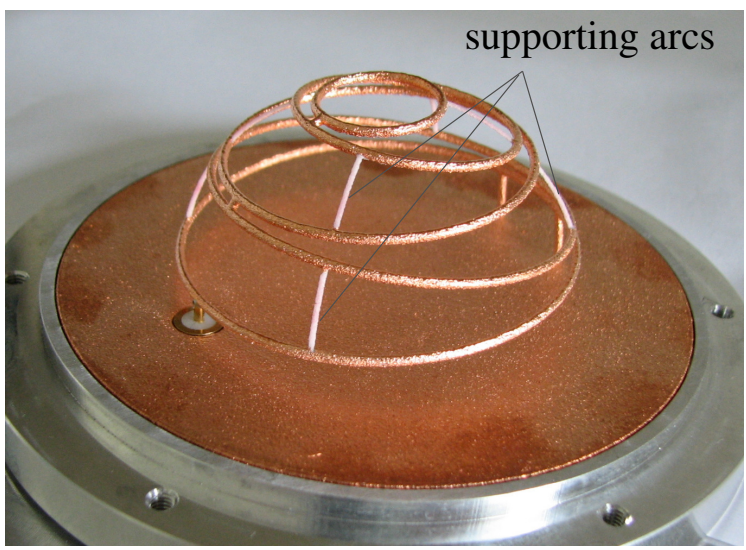

(b)

Fig. 4. Fabricated prototypes of the folded spherical helix antenna (a) and the spherical zigzag antenna (b). Supporting arcs are not coated with copper.

\section{CONCLUSION}

A novel electrically small spherical zigzag antenna is presented and investigated both numerically and experimentally. The antenna is of the electric dipole type, i.e., it radiates the $\mathrm{TM}_{10}$ spherical mode, with characteristics very similar to those of the well-known folded spherical helix antenna. The main difference is in the cross-polarization pattern, which exhibits a null in the antenna symmetry plane.

It is also shown that the modern rapid prototyping technology can be successfully applied for quick fabrication of electrically small wire antennas. The test results presented for the folded spherical helix antenna and spherical zigzag antenna illustrate that the selective laser sintering is able to reproduce fine wire structures with sufficient accuracy. The subsequent conductive coating of the printed dielectric models requires special attention, as electrically small antennas are very sensitive to the dissipation losses in their structures. It was found that coating with polycrystalline copper resulted in a much better radiation efficiency of the antennas compared to coating with conductive copper paint. The measurement results for both fabricated antenna prototypes are in good agreement with the predictions. 


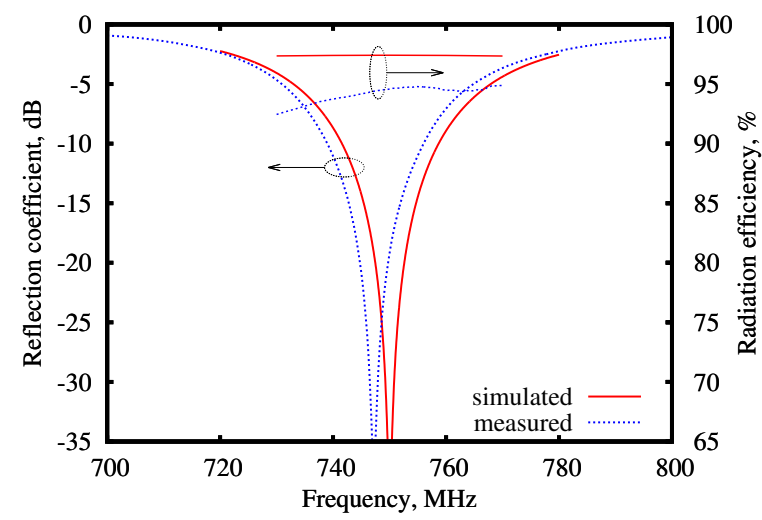

(a)

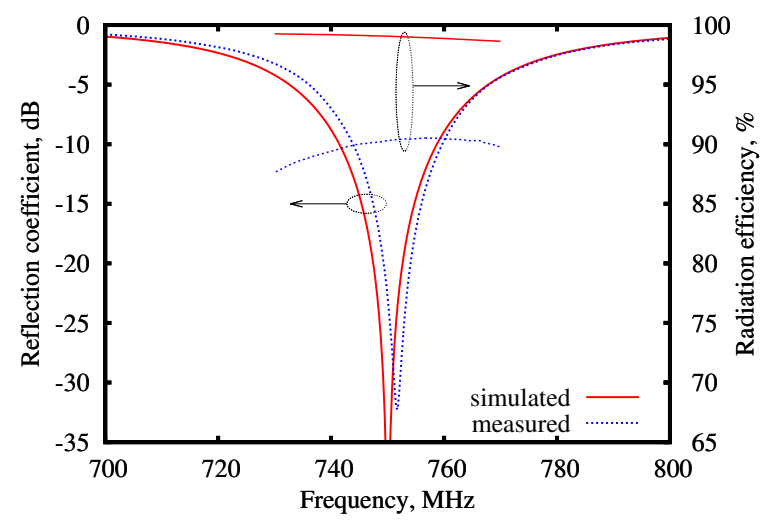

(b)

Fig. 5. Simulated (assuming solid copper wires) and measured reflection coefficients and radiation efficiency for the fabricated prototypes of the folded spherical helix antenna (a) and the spherical zigzag antenna (b).

\section{ACKNOWLEDGMENT}

Mr. Martin Nielsen is acknowledged for assembling the antennas, Dr. Sergey Pivnenko for measuring them.

\section{REFERENCES}

[1] L. J. Chu, "Physical limitations of omni-directional antennas," J. Appl. Phys., vol. 19, no. 12, pp. 1163-1175, 1948.

[2] R. Collin and S. Rothschild, "Evaluation of antenna Q," IEEE Trans. Antennas Propagat., vol. 12, no. 1, pp. 23-27, Jan. 1964.

[3] H. A. Wheeler, "The spherical coil as an inductor, shield, or antenna," Proc. IRE, vol. 46, no. 9, pp. 1595-1602, 1958.

[4] R. C. Hansen and R. E. Collin, "A new Chu formula for Q," IEEE Antennas Propagat. Mag., vol. 51, no. 5, pp. 38-41, 2009.

[5] O. S. Kim and O. Breinbjerg, "Lower bound for the radiation Q of electrically small magnetic dipole antennas with solid magnetodielectric core," IEEE Trans. Antennas Propagat., vol. 59, no. 2, pp. 679-681, February 2011.

[6] _ - "Reaching the Chu lower bound on Q with magnetic dipole antennas using a magnetic-coated PEC core," IEEE Trans. Antennas Propagat., vol. 59, no. 8, pp. 2799-2805, August 2011.

[7] O. S. Kim, "Electric dipole antennas with magnetic-coated PEC cores: Reaching the Chu lower bound on Q," IEEE Trans. Antennas Propagat., vol. 60, no. 3, pp. 1616-1619, 2012.

[8] — "Minimum Q electrically small antennas," IEEE Trans. Antennas Propagat., vol. 60, no. 8, pp. 3551-3558, 2012.

[9] S. R. Best, "The radiation properties of electrically small folded spherical helix antennas," IEEE Trans. Antennas Propagat., vol. 52, no. 4, pp. 953 960, Apr. 2004.

[10] H. R. Stuart, H. R. Stuart, and C. Tran, "Small spherical antennas using arrays of electromagnetically coupled planar elements," IEEE Antennas and Wireless Propagat. Lett., vol. 6, pp. 7-10, 2007.

[11] O. S. Kim, "Low-Q electrically small spherical magnetic dipole antennas," IEEE Trans. Antennas Propagat., vol. 58, no. 7, pp. 2210-2217, July 2010.

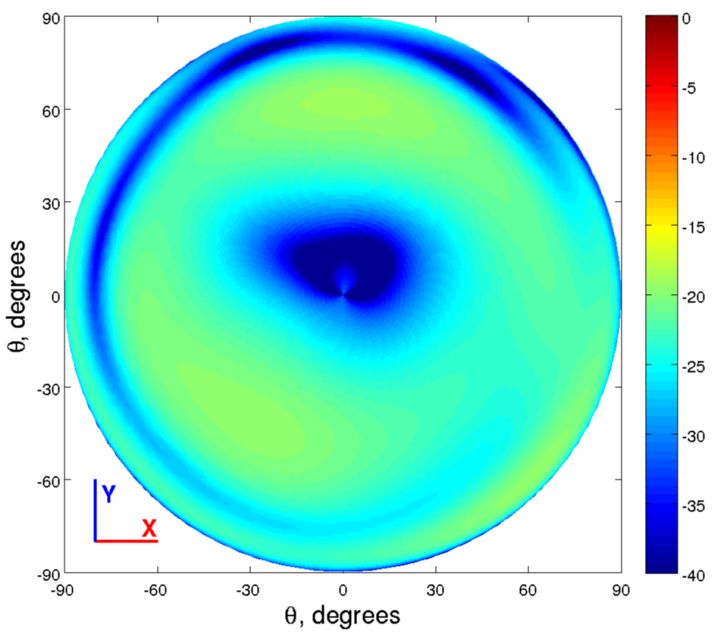

(a)

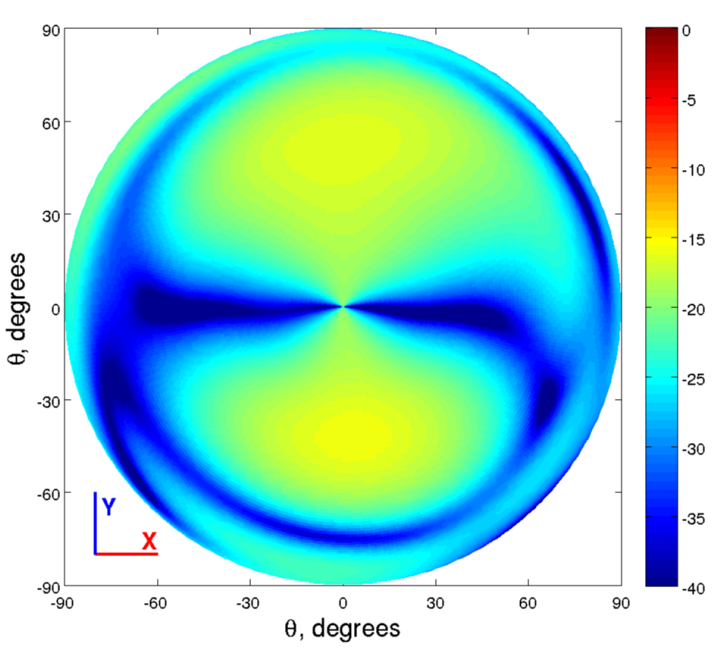

(b)

Fig. 6. Measured cross-polarization patterns for the fabricated prototypes of the folded spherical helix antenna (a) and the spherical zigzag antenna (b).

[12] J. J. Adams, E. B. Duoss, T. F. Malkowski, M. J. Motala, B. Y. Ahn, R. G. Nuzzo, J. T. Bernhard, and J. A. Lewis, "Conformal printing of electrically small antennas on three-dimensional surfaces," Adv. Mater., vol. 23, no. 11, pp. 1335-1340, 2011.

[13] J. J. Toriz-Garcia, J. J. Cowling, G. L. Williams, Q. Bai, N. L. Seed, A. Tennant, R. McWilliam, A. Purvis, F. B. Soulard, and P. A. Ivey, "Fabrication of a 3D electrically small antenna using holographic photolithography," J. Micromech. Microeng., vol. 23, no. 5, p. 055010, May 2013.

[14] C. Pfeiffer, X. Xu, S. R. Forrest, and A. Grbic, "Direct transfer patterning of electrically small antennas onto three-dimensionally contoured substrates," Adv. Mater, vol. 24, no. 9, pp. 1166-1170, 2012.

[15] M. Jobs, K. Hjort, A. Rydberg, and Z. Wu, "A tunable spherical cap microfluidic electrically small antenna," Small, Apr. 2013.

[16] T. V. Hansen, O. S. Kim, and O. Breinbjerg, "Stored energy and quality factor of spherical wave functions - in relation to spherical antennas with material cores," IEEE Trans. Antennas Propagat., vol. 60, no. 3, pp. 1281-1290, 2012.

[17] A. K. Kamrani and E. A. Nasr, "Rapid prototyping," in Engineering Design and Rapid Prototyping. Boston, MA: Springer US, 2009, pp. 339-354.

[18] O. S. Kim, "Novel electrically small spherical electric dipole antenna," in Proc. of International Workshop on Antenna Technology (iWAT2010), Lisbon, Portugal, 2010.

[19] A. Ludwig, "The definition of cross polarization," IEEE Trans. Antennas Propagat., vol. AP-21, no. 1, pp. 116-119, 1973.

[20] O. S. Kim, "3D printing electrically small spherical antennas," in Proc. IEEE Antennas Propag. Soc. Int. Symp., Orlando, Florida, USA, 7-13 July 2013, pp. 776-777. 